\title{
Survival Curves and Behavioral Profiles of Female 3xTg-AD Mice Surviving to 18-Months of Age as Compared to Mice with Normal Aging
}

\author{
Virginia Torres-Lista ${ }^{\mathrm{a}, \mathrm{b}}$, Mónica De la Fuente ${ }^{\mathrm{c}, \mathrm{d}}$ and Lydia Giménez-Llort ${ }^{\mathrm{a}, \mathrm{b}, *}$ \\ ${ }^{\mathrm{a}}$ Institut de Neurociències, Universitat Autònoma de Barcelona, Barcelona, Spain \\ ${ }^{\mathrm{b}}$ Department of Psychiatry and Forensic Medicine, Universitat Autònoma de Barcelona, Barcelona, Spain \\ ${ }^{\mathrm{c}}$ Department of Animal Physiology II, Universidad Complutense de Madrid, Madrid, Spain \\ ${ }^{\mathrm{d}}$ Research Institute Hospital 12 de Octubre, Madrid, Spain
}

\begin{abstract}
New evidence reveals a high degree of heterogeneity in Alzheimer's disease (AD) clinical and temporal patterns, supporting the existence of several subgroups of patients. Prognosticators of end-of-life dementia specific to elderly patients are necessary to address this heterogeneity. Among 3xTg-AD mice, a widely-used model for AD, a very small number of animals overcome advanced neuropathological stages of disease beyond 18 months of age. They are usually females, which reach longevity in spite of worse neuropathological status as compared to males (the morbidity/mortality paradox). We posit that 3xTg-AD long-term survivors could serve to model end-of-life dementia but also aware about the mortality selection bias. In the present study, we performed behavioral and functional phenotype in long-term survivors, 18-month-old female 3xTg-AD mice and age-matched wildtype undergoing normal aging. Animals were followed up until natural death to correlate survival with phenotype assessments. Strong similarity of their behavioral profiles in all the variables analyzed (e.g. reflexes, sensorimotor functions, locomotion, exploration, emotionality, and anxiety-like behaviors) was found, with the exception of memory impairment, which was a salient trait in old 3xTg-AD survivors. The two groups showed similar mean life expectancy and had behavioral correlates among lifespan, neophobia and long-term memory in common, with some distinctions in 3xTg-AD, supporting recent studies in end-of-life patients. In spite of the small sample size, this brief report presents an interesting scenario to further study heterogeneity and survival in Alzheimer's disease. 3xTg-AD survivors may be a model to gain insight into the frailty/survival paradigm in normal and pathological aging.
\end{abstract}

Keywords: Alzheimer's disease, animal model, behavioral correlates, end-of-life, frailty, heterogeneity, survivorship

\section{INTRODUCTION}

The 3xTg-AD mice are homozygous for the familial Alzheimer's disease (AD) mutations PS1/M146V

\footnotetext{
*Correspondence to: Lydia Giménez-Llort, PhD, Translational Behavioral Neuroscience Lab, Unitat de Psicologia Mèdica, Dept. Psiquiatria i Medicina Legal, Facultat de Medicina, Universitat Autònoma de Barcelona; Avinguda Can Domènech, Edific M, s/n, Campus Bellaterra, Cerdanyola del Vallès, 08193 Barcelona, Spain. Tel.: +34 93 5812378; Fax: +34 93 5811435; E-mail: lidia. gimenez@uab.cat.
}

and APPSwe, as well as the tauP301L human transgene [1]. These mice progressively develop the temporal- and regional-specific neuropathological patterns observed in the human $\mathrm{AD}$ brain, and mimic an important number of hallmarks of the disease [1-3]. The mortality of male and female $3 \times \mathrm{Tg}-\mathrm{AD}$ mice at 15 months of age, which is an advanced stage of amyloid and tau neuropathology, is higher than in non-transgenic (NTg) counterparts with the same genetic background $(\mathrm{C} 57 \mathrm{BL} / 6 \times 129)$ [4]. In spite of female $3 \mathrm{xTg}-\mathrm{AD}$ mice exhibiting worse 
neuropathological status than males [5], the mortality rate is more severe in male $3 \times \mathrm{Tg}-\mathrm{AD}$ mice, ranging from $33 \%$ [4] up to $100 \%$ [6] at 15 months of age. More recently, increased mortality has also been reported in the heterozygous $3 \times \mathrm{Tg}$-AD mice bred from JAX stocks as compared to the wildtype strain [7]. Interestingly, since the establishment of 3xTg-AD and NTg mouse colonies in our laboratory a decade ago [2], we have repeatedly observed that survivors beyond 15 months show a milder impairment of cognitive and emotional patterns than expected for their neuropathological status. Most of these animals overcoming the 15 month of age, usually become long-lived animals. In agreement with previously observed sex-differences found in mortality rates [3, $4,8]$, the long-term survivors are mainly females. This finding may reveal heterogeneity among $3 \times \mathrm{Tg}$ $\mathrm{AD}$ mice that manifests in advanced aging.

Heterogeneity in the progression of clinical symptoms in AD has recently been recognized as a problem in public health planning $[9,10]$. Dementia is associated with increased mortality in comparison with aged control populations [11-21]. Hence, predictors of risk of institutionalization and mortality are sought by geriatricians in order to improve longterm patient care and public health planning [15-17]. For that purpose, longitudinal follow-up studies of population-based cohorts have been conducted both in residential and nursing home settings [11-13, 19] as well as in community-dwelling older people living with dementia [i.e. 21]. In the case of $\mathrm{AD}$, the prognostic indicators of the temporal course of the disease and mortality are sought by means of prospective observational studies in persons diagnosed with 'probable $\mathrm{AD}$ ' [i.e. 15, 16] along with systematic reviews and meta-analysis [i.e. 9, 22, 23]. In patients, comorbidity, functional disability and demographic characteristics such as older age, male gender, and low education are identified as those increasing the risk of death [14]. In more recent studies, measures of disease severity at the time of diagnosis have been reported as the variables most strongly associated with mortality [9]. However, in long-term studies focusing on elderly patients and their end-of-life dementia, new evidence reveals a high degree of heterogeneity in $\mathrm{AD}$ clinical and temporal progression patterns [10]. Such heterogeneity makes it difficult to find concordance in the literature regarding prognostic tools to identify end-of-life dementia stages, that is, prognosticators of 6-month mortality, which would improve the palliative care for elderly people at advanced stages of disease [9]. Rising international concerns thus point to the need to identify reliable, sensitive, and specific prognosticators of end-of-life dementia stages [9]. As a result, important efforts have been done that talk in favor of the sensitivity of some scales, such as ADEPT score [11], HELP index [12] or the Frailty Index [13], to reliably predict mortality.

At the translational level, in the present study, we sought to characterize the behavioral profile of longterm 3xTg-AD survivors, in order to 1) confirm those personal observations related to behavioral convergence in very old animals, and 2) gain insight into prognosticators of end-of-life dementia-like stages in this animal model. For these purposes, we followed up two contemporaneous sets of middle aged (12 months of age) $3 \times \mathrm{Tg}-\mathrm{AD}$ and NTg mice until natural death. Fourteen animals surviving to 18 months of age were behaviorally assessed and thereafter, their behavioral phenotype was correlated to their survival.

\section{MATERIALS AND METHODS}

\section{Animals}

A set of 14 female mice from the Spanish colonies of homozygous 3xTg-AD $(n=7)$ and $\mathrm{NTg}(n=7)$ mice [4], genetically engineered at the University of California Irvine [1], were followed up from 12 months of age until natural death. Animals were bred, maintained and assessed at the Behavioral Facility Core of the Universitat Autònoma de Barcelona. Subjects were housed in Macrolon cages $(35 \times 35 \times 25 \mathrm{~cm})$ in groups of 3-4, per sex and genotype, under standard laboratory conditions of food (SAFE A04, Panlab, S.L.) and water ad libitum, $22 \pm 2^{\circ} \mathrm{C}$, a $12 \mathrm{~h}$ light:dark cycle, and relative humidity of 50-60\%. Genotypes were regularly confirmed by RT-PCR analysis. Behavioral assessments were performed at 18 months of age, blind to the experimenter, in a counterbalanced manner, under approval of local policy (481CEEAH/8700 Generalitat de Catalunya), in accordance with Spanish legislation and the EU Directive (2010/63/UE) on "Protection of Animals Used for Experimental and Other Scientific Purposes". Two NTg mice and one 3xTg-AD mice showed impaired navigation capacities and were excluded of the water maze test and of subsequent Pearson's correlation analysis. Therefore, the survival curves include the 14 animals, while the final size sample in the behavior was five NTg mice and 
six $3 \times$ Tg-AD mice. The study complies with the ARRIVE guidelines developed by the NC3Rs [24].

\section{Behavioral assessments}

At 18 months of age, a comprehensive screening of several physical, emotional, and cognitive functions was successively performed using a battery of 10 tests based on three main behavioral dimensions [4] that can be described as follows:

\section{1) Physical status, reflexes, and sensorimotor functions}

The body weight of animals was monitored as an index of health/frailty status. Reflexes (visual reflex and posterior leg extension reflex tests) were measured three times by holding the animal by the tail and slowly lowering it to a black surface. Equilibrium, coordination, strength, and resistance were assessed in the rod and hang tests using two levels of difficulty in each test (wood versus wire rod; $5 \mathrm{~s}$ versus $60 \mathrm{~s}$ trials in the hang test).

Motor coordination and equilibrium were assessed by the distance covered and the latency to fall off a horizontal wooden rod ( $1.3 \mathrm{~cm}$ wide) on two consecutive $20 \mathrm{~s}$ trials, respectively. In order to increase the difficulty of the task, the test was repeated on a metal wire rod $(1 \mathrm{~cm}$ diameter). Thereafter, motor abilities were measured again in the wire hang test, a more demanding task which consists in allowing the animal to cling from the middle of a horizontal wire (diameter: $2 \mathrm{~mm}$, length: $40 \mathrm{~cm}$, divided into eight $5 \mathrm{~cm}$ segments) with the forepaws for two trials of $5 \mathrm{~s}$ and a third $60 \mathrm{~s}$ trial. The first two trials are also considered training trials. Motor coordination was measured as the mean distance covered in the two $5 \mathrm{~s}$ trials and the distance covered in the $60 \mathrm{~s}$ trial. Muscle strength and resistance were measured as the mean time until falling off the wire in the two $5 \mathrm{~s} \mathrm{tri-}$ als and the $60 \mathrm{~s}$ trial, respectively. All the apparatuses were suspended $40 \mathrm{~cm}$ above a padded table.

\section{2) 'Behavioral and Psychological Symptoms of Dementia' (BPSD)-like behaviors}

Changes in emotionality, increased neophobia and other signs of anxiety-like responses, all of them BPSD-like behaviors modeled in 3xTg-AD mice [2], were measured in classical unconditioned tests. The tests evaluate locomotion/exploration, anxietylike behaviors, and emotionality under three different anxiogenic conditions: mild neophobia in a new home-cage (Corner test), direct exposure to an open and illuminated field (Open-field), and black corridors of a maze resembling burrows (T-maze test).

\section{Corner test}

Neophobia to a new home-cage (Macrolon, $35 \times 35 \times 25 \mathrm{~cm}$, with beddings) was assessed by introducing the animal into the center of the cage and counting the number of visited corners and rearings during a period of $30 \mathrm{~s}$. Latency of the first rearing was also recorded.

\section{Open-field test}

The mouse was placed in the center of the apparatus (home-made, wooden, white, $50 \times 50 \times 25 \mathrm{~cm}$ high) and observed for $5 \mathrm{~min}$. Horizontal (crossings of $10 \times 10 \mathrm{~cm}$ squares) and vertical (rearings) locomotor activities were recorded for each minute of the test. We also recorded the latency of the sequence of the following behavioral events: initial freezing (latency of initial movement), thigmotaxis or discrimination of unprotected/protected areas in the test (latency of leaving the central $10 \times 10 \mathrm{~cm}$ square and that of entering in the peripheral ring $10 \mathrm{~cm}$ to the walls), and self-grooming behavior (latency, number, and duration of groomings). Defecation was also measured.

\section{T-Maze Test}

The spontaneous exploratory behavior of mice was tested in a T-shaped maze (with arms $25 \mathrm{~cm}$ in length). The animal was placed inside the 'vertical' arm of the maze with its head facing the end wall. The performance was evaluated by determining with a chronometer the time elapsed until the animal crossed (four paws criteria) the intersection of the three arms. Defecation was also measured.

\section{3) Cognitive function}

Cognitive function was assessed in three paradigms of 'learning and memory' of increasing difficulty in the Morris water maze: a cue learning task of a visible platform to assess visual perceptual learning and memory, followed $24 \mathrm{~h}$ later by a place learning task using a hidden platform in a reversed position to assess spatial reference learning and memory, and a final trial for long-term memory where the platform had been removed. During each trial of the learning tasks, the escape latency was measured by means of a computerized tracking system (SMART, Panlab S.A., Barcelona, Spain). 


\section{Visual perceptual learning task}

On days 1-2 (Cue 1 and Cue 2), animals were tested for visual perceptual learning and memory. Mice were trained to locate a platform $(7 \mathrm{~cm}$ diameter, NW position) elevated $1 \mathrm{~cm}$ above the water level with its position indicated by a visible striped flag $(5 \times 8 \times 15 \mathrm{~cm})$ in a circular pool (Intex Recreation Corp. CA, USA; $91 \mathrm{~cm}$ diameter, $40 \mathrm{~cm}$ height, $25^{\circ} \mathrm{C}$ opaque water). The cue learning task consisted of four trials $20 \mathrm{~min}$ apart per day (ITT, intertrial time), during two consecutive days. In each trial, the mouse was gently released (facing the wall) from a randomly selected starting point (N, S, E, or W), and was allowed to swim until it escaped onto the platform.

\section{Place learning task}

On days 3-7, five place tasks (PT1-PT5) for spatial reference learning and memory were administered, followed by one probe trial, usually called 'removal' (RM) for long-term memory. In the PTs, the hidden platform was always located in a reversed position (the middle of the SE quadrant) and the mouse was trained to locate the platform thanks to four distal visual cues. As before, the acquisition task consisted of four trial sessions per day, with $20 \mathrm{~min}$ of ITT. In both tasks, mice that failed to find the platform within $60 \mathrm{~s}$ were placed on it for $10 \mathrm{~s}$, the same period allowed for the successful animals.

\section{Removal}

On day 8 , the platform was removed from the maze and the mouse performed a probe trial of $60 \mathrm{~s}$. The computerized system allowed to measure the time spent swimming in the Place Task trained platform quadrant of the pool $(\mathrm{P})$, adjacent right quadrant (Ar), opposite quadrant $(\mathrm{O})$, and adjacent left $(\mathrm{Al})$.

\section{Longitudinal assessment of survival}

Survival was monitored daily, from 12 months of age (week 52) until natural death. Correlations between life span (age in days at death) and behavioral variables were searched for in all the pool of animals and independently for each genotype.

\section{Statistical analysis}

SPSS 15.0 software (SPSS, Chicago, IL, USA) was used for the statistical analysis of the results. The data are expressed as mean \pm SEM or frequency. Differences between two independent groups were evaluated with Student's $t$-test. In the temporal courses, RMA, Repeated measures ANOVA, followed by Duncan's post-hoc comparison was used for within-subject analysis. Behavioral correlations with life span were analyzed with Pearson's correlation. Differences in life span were studied through the Kaplan-Meier test. Statistical significance was considered at $p<0.05$.

\section{RESULTS}

\section{Similar physical and sensorimotor functions in 18-month-old 3xTg-AD and NTg mice}

As detailed in Table 1 body weights, reflexes and sensorimotor functions of 18-month-old 3xTg-AD mice did not differ from those observed in NTg mice with normal aging (genotype effects, Student $t$-test, $p>0.05$ or even equal values) except for those tests were their freezing behaviors resulted in a longer latency to fall (the wood rod test and $60 \mathrm{~s}$ trial of the hanger test). In the wire rod and hang tests, and independently of genotype, animals did not cover any distance and latencies were very short. In the hanger test, a few animals performed the trial of $60 \mathrm{~s}$. In both groups, the measures of resistance and coordination revealed high individual variability.

\section{Striking similarities in BPSD-like symptoms and emotional behaviors in 18-month-old 3xTg-AD and NTg mice}

One of the advantages of 3xTg-AD mice as a model of $\mathrm{AD}$ is that they exhibit altered exploration and emotionality and show increased anxiety-like behaviors, resembling some of the BPSD symptoms reported in human patients. Unexpectedly, results in the corner test, open-field test, and T-maze evidenced striking similarities between 18-month-old 3xTg-AD and NTg mice. Thus, all the analyzed behavioral variables (see Table 1 and Fig. 1), including those related to the time course of elicitation of behavioral events and emotionality, as well as the measures of neophobia in the corner test, exploration in the open-field test, and coping with stress strategies in the T-maze, showed convergence of profiles. Furthermore, as observed in the physical and sensorimotor aspects, both groups of mice showed high individual variability that resulted in high statistical variance. 
Table 1

Similarity of physical, sensorimotor, exploratory and BPSD-like domains, and salience of cognitive deficits in 18-month-old female 3xTg-AD mice as compared to sex- and age-matched NTg mice with normal aging

\begin{tabular}{|c|c|c|c|}
\hline & \multicolumn{2}{|c|}{ Females, 18-month-old } & \multirow[t]{2}{*}{ Genotype differences } \\
\hline & $\begin{array}{c}\text { NTg mice } \\
n=5\end{array}$ & $\begin{array}{c}3 \times \mathrm{Tg}-\mathrm{AD} \text { mice } \\
n=6\end{array}$ & \\
\hline \multicolumn{4}{|c|}{ 1. Physical status, reflexes and sensorimotor function } \\
\hline Body weight $(\mathrm{g})$ & $34.20 \pm 2.20$ & $36.27 \pm 2.38$ & n.s. \\
\hline Visual placing reflex (3 trials) & $3 / 3$ & $3 / 3$ & equal \\
\hline Posterior leg reflex (3 trials) & $3 / 3$ & $3 / 3$ & equal \\
\hline \multicolumn{4}{|l|}{ Wood rod test (two 20-s trials) } \\
\hline Equilibrium (mean falling latency, s) & $9.80 \pm 2.71$ & $20 \pm 0$ & $* *$ \\
\hline Coordination (mean distance, $\mathrm{cm}$ ) & $0 \pm 0$ & $0 \pm 0$ & equal \\
\hline \multicolumn{4}{|l|}{ Wire rod test (two 20-s trials) } \\
\hline Equilibrium (mean falling latency, s) & $0.20 \pm 0.20$ & $5.33 \pm 2.88$ & n.s. \\
\hline Coordination (mean distance, $\mathrm{cm}$ ) & $0 \pm 0$ & $0 \pm 0$ & equal \\
\hline \multicolumn{4}{|l|}{ Wire hang test (two 5 -s trials) } \\
\hline Strength (mean time hold, s) & $2.40 \pm 0.93$ & $3.83 \pm 0.75$ & n.s. \\
\hline Coordination (mean distance, segments) & $0 \pm 0$ & $0 \pm 0$ & equal \\
\hline \multicolumn{4}{|l|}{ Wire hang test (one 60-s trial) } \\
\hline Resistance (time hold, s) & $3.60 \pm 1.81$ & $40.67 \pm 12.23$ & $*$ \\
\hline Coordination (distance, segments) & $0 \pm 0$ & $1.33 \pm 0.61$ & n.s. \\
\hline \multicolumn{4}{|c|}{ 2. BPSD-like behaviors and exploratory activity } \\
\hline & & & \\
\hline Vertical activity (latency, s) & $14.60 \pm 3.04$ & $21.17 \pm 2.91$ & n.s. \\
\hline Vertical activity (number) & $1.80 \pm 0.37$ & $1.33 \pm 0.42$ & n.s. \\
\hline Horizontal activity (number) & $8.20 \pm 1.39$ & $6.17 \pm 1.33$ & n.s. \\
\hline \multicolumn{4}{|l|}{ Open field test ( $5 \mathrm{~min})$} \\
\hline Initial freezing (latency, s) & $3.80 \pm 1.85$ & $25.33 \pm 20.35$ & n.s. \\
\hline Exit of the center (latency, s) & $10.80 \pm 3.02$ & $33.00 \pm 20.01$ & n.s. \\
\hline Entrance to the periphery (latency, s) & $61.80 \pm 50.37$ & $94.83 \pm 31.78$ & n.s. \\
\hline Vertical activity (latency, s) & $121.80 \pm 46.56$ & $173.00 \pm 27.30$ & n.s. \\
\hline Vertical activity (number) & \multicolumn{2}{|c|}{ See Fig. 1} & n.s. \\
\hline Horizontal activity (number) & \multicolumn{2}{|c|}{ See Fig. 1} & n.s. \\
\hline Self-grooming (latency, s) & $147.00 \pm 27.51$ & $212.50 \pm 29.09$ & n.s. \\
\hline Self-grooming (number) & $2.00 \pm 0.45$ & $0.83 \pm 0.31$ & n.s. \\
\hline Defecation boli (number) & $0 \pm 0$ & $0 \pm 0$ & equal \\
\hline \multicolumn{4}{|l|}{ T-maze $(5 \mathrm{~min})$} \\
\hline Arrival at the T-intersection (latency, s) & $14.40 \pm 3.17$ & $8.50 \pm 1.09$ & n.s. \\
\hline Defecation boli (number) & $0.60 \pm 0.60$ & $0.83 \pm 0.31$ & n.s. \\
\hline \multicolumn{4}{|l|}{ 3. Lifespan } \\
\hline Median life expectancy (weeks) & $105.0 \pm 8.6$ & $103.71 \pm 3.6$ & n.s. \\
\hline
\end{tabular}

Results are expressed as means \pm SEM or incidence. Student's $t$-test, ${ }^{* *} p<0.01,{ }^{*} p<0.05, n . s . p>0.05$ versus NTg mice.

\section{Cognitive deficits are the most salient distinctive trait in 3xTg-AD mouse survivors}

Cognitive deficits in spatial reference learning and memory differentiated the 18-month-old 3xTg-AD mice from age-matched NTg counterparts. In the assessment of visual perceptual learning and memory (Fig. 2A), the two groups of mice showed the same mean escape latency on the first day of the cue task. On the second day of testing, 3xTg-AD mice showed worse performance, although it did not reach statistical significance. On Day 3, the platform was hidden and located in a reversed position; this made the new paradigm a difficultplace task. In this test, NTg females took longer to solve the problem than in their first performance in the maze with a visible and cued platform (PT1 versus CUE, $p<0.05$ ). They had to search the hidden platform. In contrast, $3 \times \mathrm{Tg}$-AD females took as long as in their first cue task. Therefore, differences between genotypes were found when comparing their ability to solve the two tasks for the first time (PT1-CUE1, $p<0.05$, inset Fig. 2A), and after two consecutive days of cue learning 


\section{EXPLORATORY ACTIVITY}
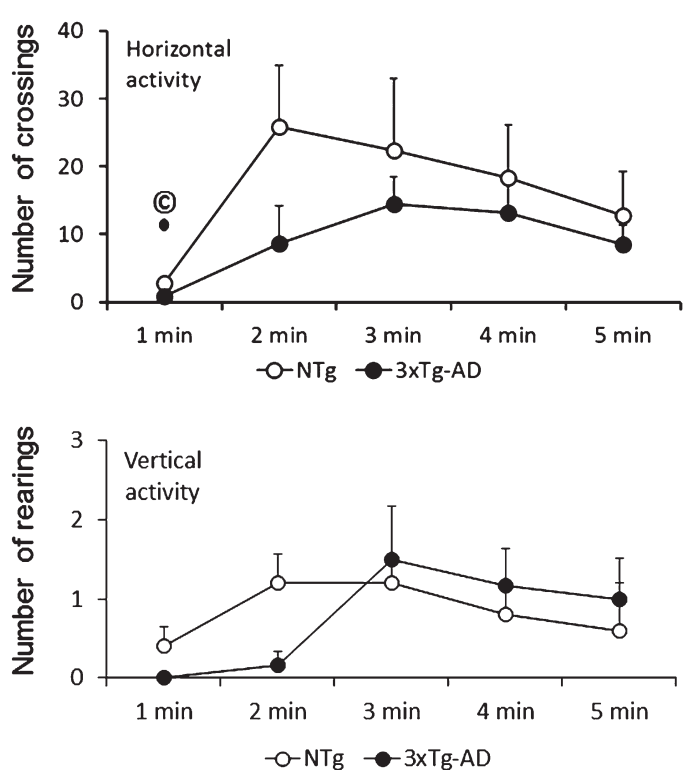
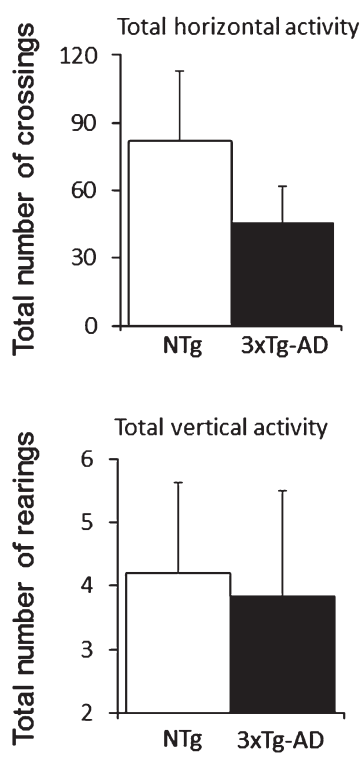

Fig. 1. Similar exploratory activity patterns and total counts exhibited in the anxiogenic open-field test in 18-month-old female $3 x$ Tg-AD mice as compared to sex- and age-matched NTg mice with normal aging. Interestingly, horizontal activity (number of crossings) shown in the first minute of the test, a variable that measures the level of neophobia to this new environment, correlated (@positive Pearson's correlation, $p<0.05$ ) with lifespan of NTg and 3xTg-AD mice (see Fig. 4B and 4J).

(PT1-CUE2, $p<0.05$, inset Fig. 2A). On the following days (PT2-PT5), spatial reference memory assessed by means of place-learning did not show any differences between genotypes, since both groups of mice exhibited similar mean latencies in finding the hidden reversed platform. Twenty-four $h$ after the fifth day of place learning, an extra trial with removal of the platform indicated bad performance, with significant preference for the opposed quadrant, in both genotypes. In view of the results, and in order to confirm the level of memory of the trained position, the latency of reaching the previous location was also recorded. Both groups of mice reached the trained location in less than 10 seconds, but thereafter invested most of the time in the wrong quadrant of the maze.

\section{Behavioral correlates between survival and cognitive measurements}

Survival curves (Fig. 3) of the sample of 14 females, seven mice with $3 \times \mathrm{Tg}$-AD genotype and seven NTg mice, as recorded since 12 months of age to natural death, showed statistical differences due to a shorter lifespan in the transgenic group (3xTg-AD: 120 weeks versus NTg: 140 weeks). However, the groups did not differ in their median life expectancy (3xTg-AD: $103.71 \pm 3.60$ weeks versus NTg: $105.0 \pm 8.50)$.

The search for significant correlations between lifespan and performance in the 10 behavioral tests (see Fig. 4) pointed to the consistency of sensorimotor function and cognition as the best end-of-life prognosticators (Pearson's correlation, $p<0.01$ ) in the sample of old NTg mice $(n=5)$ and $3 \times$ Tg-AD mice $(n=6)$, respectively. In particular, survival of NTg was strongly correlated with equilibrium (Fig. 4E, latency of falling in the wood rod test, $R=0.977$ ), while long-term memory exhibited in the removal test (Fig. 4K, latency to arrive to the previous platform location, $\mathrm{R}=-0.949$; Fig. $4 \mathrm{~L}$, time spent into the $\mathrm{Ar}$ quadant, $\mathrm{R}=0.918$ ) by old $3 \times \mathrm{Tg}$-AD mice surviving to advanced stages of disease were the variables showing a strong correlation with lifespan. Besides, short and long-term memory in the NTg mice (Fig. 4F, $\mathrm{R}=0.929$; Fig. 4G, $\mathrm{R}=0.895$; Fig $4 \mathrm{H}$, $\mathrm{R}=0.915$ ) and the level of neophobic-like behaviors exhibited in the corner and open-field tests by the $3 \times \mathrm{Tg}-\mathrm{AD}$ mice (Fig. 4I, R=0.815; Fig. 4J, $\mathrm{R}=0.812$ ) showed good Pearson's correlations $(p<0.05)$ with their respective lifespans. Finally, when both genotypes were analyzed together, the level of neophobia shown in the T-maze (Fig. 4A. $\mathrm{R}=0.678$ ) and the open-field (Fig. $4 \mathrm{~B}, \mathrm{R}=0.679$ ) 


\section{A LONG-TERM LEARNING AND MEMORY}

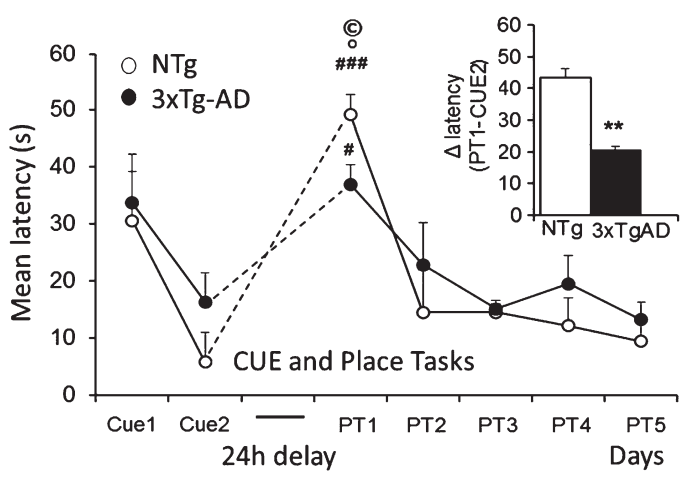

B SHORT- AND LONG-TERM LEARNING AND MEMORY

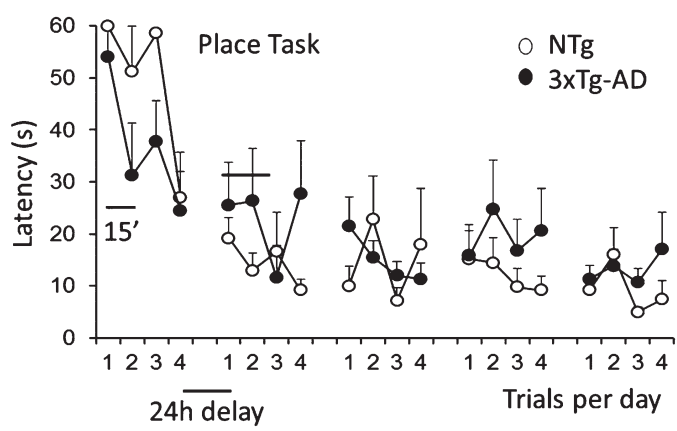

\section{LONG-TERM MEMORY}

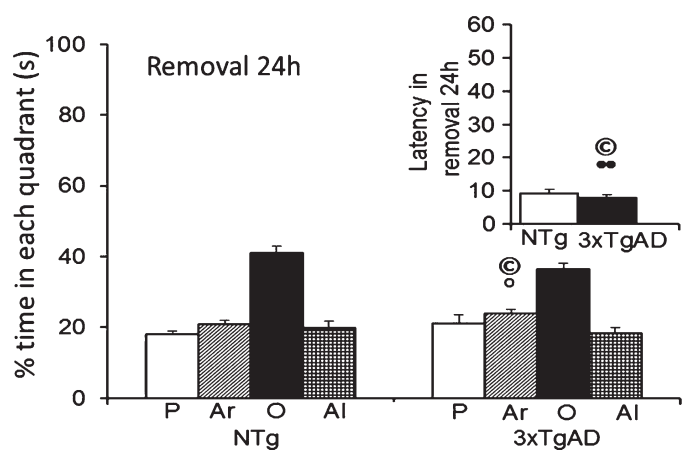

Fig. 2. Salient learning and memory deficits in three paradigms of the Morris water maze in 18-month-old female 3xTg-AD mice surviving to advanced stages of disease as compared to age-matched NTg counterparts. A) The cue and place tasks showed similar acquisition curves over days, but genotype differences could be found when the platform was hidden and located in a reversed position. The new task was more difficult for animals remembering the prior location, so it took them more time to find the new position. B) Detailed analysis of short- and long-term memory assessed in the place tasks evidenced that cognitive impairment was the salient trait for distinguishing the genotypes. C) Memory, assessed in the Removal, showed lack of preference for the trained quadrant in both groups pf mice, with a slightly worse performance in the $3 \mathrm{xTg}$-AD mice. Student's $t$-test, ${ }^{*} p<0.05,{ }^{* *} p<0.01$ versus NTg mice. Paired $t$-test ${ }^{\#} p<0.05,{ }^{\# \#} p<0.001$ PT1 versus Cue2. (C) Pearson's correlations between behavioral variables and lifespan: - positive, ${ }^{\circ}$ negative, $p<0.01$.

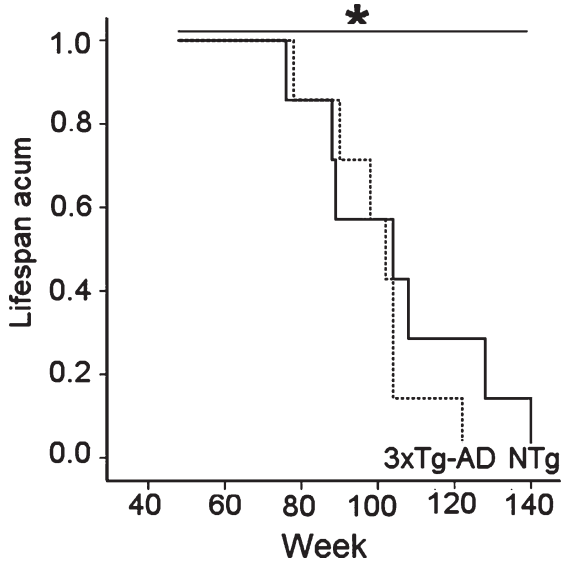

Fig. 3. Difference in the survival curves of 18-month-old female 3xTg-AD mice and age-matched NTg counterparts was found in spite of similar mean life expectancies. Kaplan-Meyer test, $* p<0.05$.

as well as the long-term memory in the removal test (Fig. 4C, $\mathrm{R}=-0.678$; Fig. 4D, $\mathrm{R}=0.727$ ) were good indicators of lifespan $(p<0.05)$.

\section{DISCUSSION}

The present study was aimed to carry out a behavioral and functional analysis of mouse phenotype in female $3 \times \mathrm{Tg}$-AD mice surviving to 18 -month-old as compared to NTg counterparts with normal aging. A second objective was to follow these animals up until natural death to find correlates between their survival and specific items of their behavioral phenotypes. Therefore, animals were presented with 10 behavioral tests assessing several functions and involving different levels of difficulty that can be categorized into three main behavioral dimensions. First, physical status and sensorimotor functions were scored, followed by evaluation of emotional and anxiety-like profiles, and finally, cognitive functions were assessed in spatial learning and memory tasks. The main findings were: 1) $3 \times \mathrm{Tg}$-AD mice that survived beyond advanced stages of disease (beyond 18 months of age) presented severe physical/sensorimotor and anxietylike profiles, but were hardly distinguishable from normal aging counterparts; 2) cognitive deficits were the salient behavioral phenotype of transgenic mice; 3) transgenic mice presented greater individual variability at 18 months than that widely reported at 12-15 months [see 2, 3, 6]; and 4) in both genotypes, neophobia and long-term memory correlated with survival; with sensorimotor function and long-term memory being the functions showing the strongest 
BEHAVIORAL CORRELATES
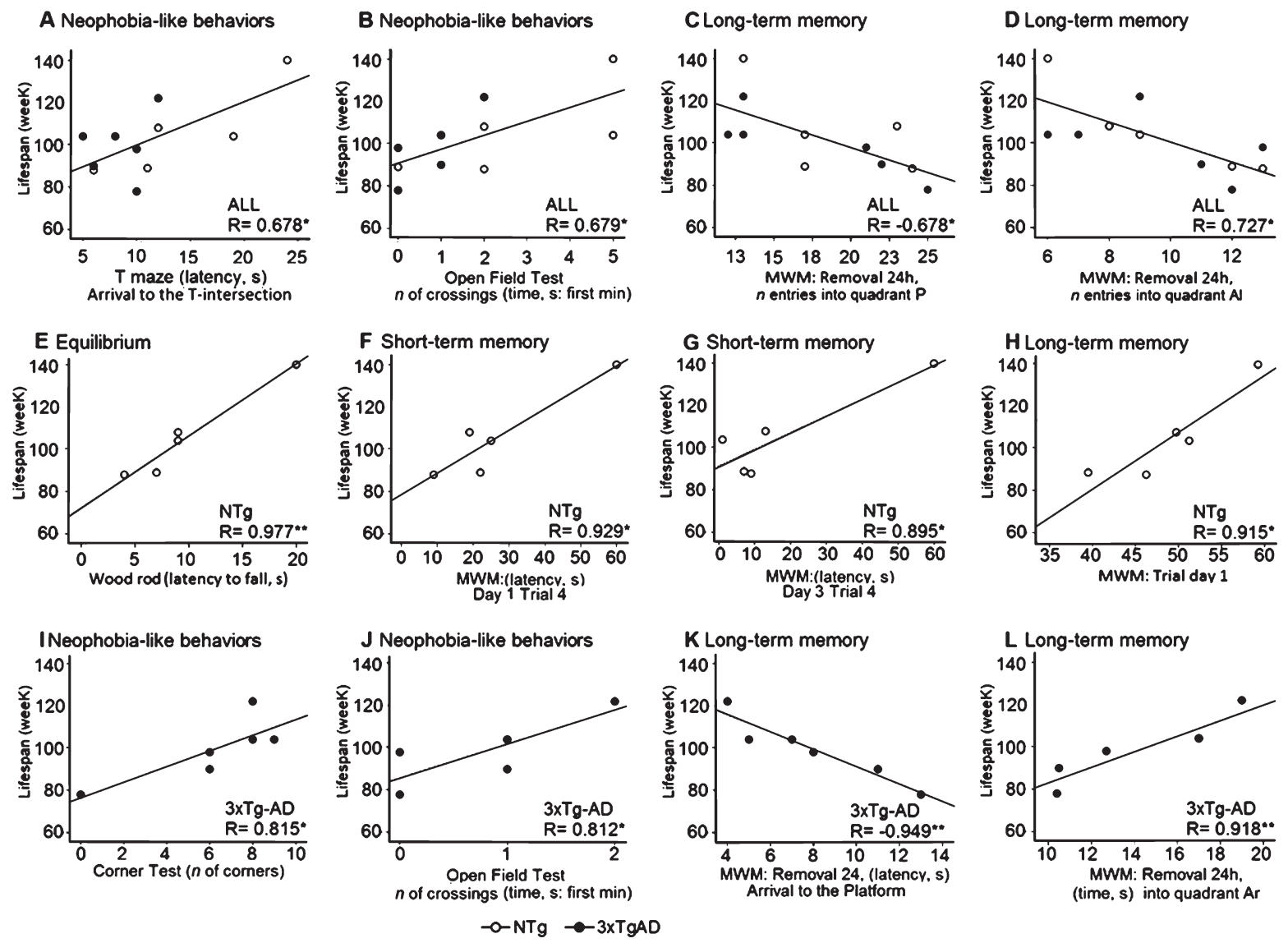

Fig. 4. In six 18-month-old female 3xTg-AD mice and five age-matched NTg counterparts, Pearson's correlations between lifespan and behaviors were studied. Correlations $(p<0.01)$ found between lifespan and long-term memory in old 3xTg-AD mice $(n=6)$, and between lifespan and sensorimotor abilities in old NTg mice $(n=5)$. Correlation $p<0.05$ was also found between lifespan and neophobia as assessed in the corner- and open-field tests (in the $3 \mathrm{xTg}$-AD mice) but also in the T-maze (all sample, $n=11$ ). Besides, long-term memory correlates showed a good consistency, since they also showed correlations $p<0.05$ with lifespan in the old NTg mice $(n=5)$ and when the whole sample was analyzed $(n=11)$. Short-term memory was found to be correlated with lifespan in the sample of NTg mice $(n=5)$. Further studies in a larger sample size are needed. (@) Pearson’s correlations between behavioral variables and lifespan: ${ }^{\bullet}$ positive, ${ }^{\circ}$ negative, $p<0.01$.

correlations with lifespan in old NTg and 3xTgAD mice, respectively. The general similarity of behavioral and functional decline in 3xTg-AD and non-transgenic mice with the exception of subtle memory deficits in the transgenic mice, also suggest that a subset of $3 \times \mathrm{Tg}-\mathrm{AD}$ mice, that is, those surviving to 18 months of age, may develop natural protection against disease over time. Altogether, the $3 \mathrm{xTg}-\mathrm{AD}$ survivors may provide a translational scenario for study of the heterogeneous manifestations reported in $\mathrm{AD}$ in end-of-life patients [10].

It is worth emphasizing that 18 -month-old mice survived advanced neuropathological stages of disease. The vast majority of behavioral assessments are performed in young adulthood, adults, and middle- aged animals, whose survival curves still permit experimental designs without risk to the sample size. The reduced survival of animals, the concomitant increase of laboratory costs, and the heterogeneity of the aged populations are among the practical difficulties that researchers confront and need to solve [22-25]. As a result, behavioral analyses in old and very old mice (18 months of age and more, respectively) are scarce, even more so in models of neurodegenerative diseases [e.g., 22-25]. The current recommendations emphasize the need for aging models more closely resembling the complex agerelated frail scenario of disease in humans for better translational outcomes of age-related neurodegenerative disease research $[26,27]$. The paucity of data 
increases the value of our analysis in 18-month mice, which, in the C57BL6 $\times 129$ strain, are an 'old' population according to their lifespan. In homozygous 3xTg-AD mice with a C57BL6 $\times 129$ background, severe mortality rates have been reported by our laboratory [2-4] and others [7] at 15-17 months (65-71 weeks) of age. Still, it is important to make the statement that in the present work the small sample size is a limitation of the study.

Most mice, transgenic and non-transgenic, were found to have poor physical motor abilities, as assessed in the rod and hanger tests. The severe impairment showed in the lack of coordination and in the short latencies of falling. In the wood rod test, the anxious-like profile of 3xTg-AD results in petrification that allows the animal to stay in the bar during the 20 seconds of duration of the test. No differences in genotype were observed in the corner, open-field, and T-maze tests, three classical unconditioned tests measuring neophobia, exploratory activity, and emotionality. The detailed analysis of this convergence of behavioral profiles involves considering the contribution of disease-related patterns and/or aging-related decline per se. Interestingly, it is the poor performance of the wild-type animals that best explains the convergence of behaviors of transgenic and nontransgenic mice with regards to physical, emotional, and anxiety-like performance. Accordingly, the comparison of the present results with those previously reported in 12- and 15-month old females of the same colonies - bred, maintained and assessed under identical experimental conditions - reveals a 3-4fold drop of function due to aging in both genotypes $[2,3,6]$. Thus, impoverished performance in 18female NTg mice appears to be an important part of the explanation for the observed convergence in the physical and non-cognitive behavioral patterns with those of female $3 \times \mathrm{Tg}$-AD mouse survivors. It may also contribute to the similarity found in their mean life expectancy but not in the overall life span which still remained reduced in the $3 \times \mathrm{Tg}$-AD mice as compared to NTg mice. The survival of very old $3 \times \mathrm{Tg}-\mathrm{AD}$ mice points to the existence of protective mechanisms that render a subset of transgenic mice resilient to pathology but not to normal aging.

Importantly, in spite of the overall similarity in the physical and non-cognitive profiles, impaired cognition remains the salient distinctive trait in 18month-old female $3 \times \mathrm{Tg}$-AD mice as compared to NTg mice. This difference highlights the distinct contribution of aging and disease-related components to behavioral readouts. Specifically, the results in the water maze of $3 \mathrm{xTg}-\mathrm{AD}$ mice resembled those previously reported by our laboratory in very old ( 24 months of age) wild-type animals [28], and they are in agreement with the increased rate of aging or biological age we have reported in these transgenic animals at prodromal and early stages of disease $[29,30]$. In addition, at this age the deficits in longterm memory shown in the removal confirm the lack of preference for the trained quadrant exhibited by both 12-month-old female 3xTg-AD and also NTg mice [2]. By contrast, salient behaviors in 3xTg$\mathrm{AD}$ mice were the impairment in cognitive flexibility as revealed by the difficulty in meeting new task demands (reversed place task after cue learning). These results are in accordance with the persistence of behavioral patterns described by our laboratory in 13-month-old male $3 \mathrm{xTg}$-AD mice in the water maze [25].

The present results offer an interesting experimental setting to study the morbidity/mortality paradox. The onset and increase of mortality in 3xTg-AD mice are concomitant with the establishment of amyloid- $\beta$ and tau pathologies occurring at 12 and 15 months of age, respectively [2-4]. We have repeatedly reported that the increased vulnerability of $3 \times \mathrm{Tg}-\mathrm{AD}$ mice at 15 months of age is concurrent with impairment of the neuroimmunoendocrine system, with sex-dependent profiles, leading to advanced biological age as compared to age-matched normal aging NTg counterparts $[3,4,30]$. The paradox is that homozygous female $3 \times \mathrm{Tg}$-AD mice show greater survival than males although they harbor the same transgenic genetic background, and present worse neuropathological status $[2-4,6]$. Why this happens is an outstanding question that highlights a limitation in the studies designed to explore advanced neuropathological stages of disease. We want to remark that it is not only difficult to obtain a sufficient number of animals, but it may also be the case that they do not represent the worst scenario in $\mathrm{AD}$ progression.

The longitudinal study of this cohort of 14 mice, seven $3 \times \mathrm{Tg}-\mathrm{AD}$ and seven NTg mice, across their life span from 12 months of age until natural death showed similar mean life expectancies. However, genetic differences still were found when the overall survival curves were analyzed. Correlations of lifespan with behavior indicate that predictors were mostly related to cognitive function. The correlations found with the sensorimotor abilities in the NTg mice agree with recent reports of elements of mobility as predictors of survival in elderly patients and those with dementia [31]. In our case, in the equilibrium 
bars, the freezing behavior of $3 \mathrm{xTgAD}$ mice usually results in a false positive 'long latency to fall' ceiling effect that preclude any correlation analysis. It's interesting to note that the three variables measuring neophobia (number of corners in the corner test, number of crossing in the first minute of the open-field test and the latency to reach the intersection in the T-maze) indicate that animals with a better performance (lower neophobia) were those also showing longer lifespans. Therefore, a better physical condition, ability to explore new environments and long-term memory were found as the best indicators of lifespan. In spite of the inherent limitations of the sample size of the present study, the strong behavioral correlations $(p<0.01)$ with lifespan make the present results of interest for further exploration in a larger size sample.

The scarce number of prognosticators in these long-lived patient populations may result from recently reported loss of integrative functions and behavioral derangements due to aging and/or the progress of the disease [31, 32]. Another source of heterogeneity is that long-term survivors, by definition, may have developed protective mechanisms against disease that act as an additional confounding factor in survival prognosis. Further phenotypical analysis of $3 \times \mathrm{Tg}-\mathrm{AD}$ survivors using longitudinal designs and including the quantification of rates of functional decline in different domains will help refine the translational potential of animals modeling the complex and heterogeneous phenotype of $\mathrm{AD}$ recently reported in human patients [32]. More importantly, it should shed further light on the neuronal mechanisms underlying this morbidity/mortality paradox at both the neuropathological and behavioral levels [33]. All in all, the present results point to female $3 \times \mathrm{Tg}$-AD mice and end-of-life frailty in sensorial and cognitive profiles as relevant scenarios for the study of both common and distinct mechanisms underlying survival in aging- and ADassociated functional decline.

\section{ACKNOWLEDGMENTS}

We thank Prof. Frank M. LaFerla, Institute for Memory Impairments and Neurological Disorders, University of California Irvine, CA, USA, for kindly providing the progenitors of the Spanish colonies, and Elena Galea, Institute of Neuroscience, Universitat Autònoma de Barcelona, Spain, for reading the manuscript and providing critical comments. This study was supported by Instituto de Salud Carlos III-FEDER, PI107/0283 to L.G.-L, PI15/01787 to M.D.F and RETICEF RD12/0043/0018-FEDER. Plan Nacional I+D+I 2008-2011, Instituto de Salud Carlos III-Subdirección General de Evaluación y Fomento de la Investigación, cofinanced by Fondos FEDER (the European Regional Development Fund [ERDF]).

\section{CONFLICT OF INTEREST}

The authors declare that there are no financial or commercial conflicts of interest.

\section{REFERENCES}

[1] Oddo S, Caccamo A, Shepherd JD, Murphy MP, Golde TE, Kayed R, Metherate R, Mattson MP, Akbari Y, LaFerla FM (2003) Triple-transgenic model of Alzheimer's disease with plaques and tangles: Intracellular $\mathrm{A} \beta$ and synaptic dysfunction. Neuron 39, 409-421.

[2] Giménez-Llort L, Blázquez G, Cañete T, Johansson B, Oddo S, Tobeña A, LaFerla FM, Fernández-Teruel A (2007) Modeling behavioral and neuronal symptoms of Alzheimer's disease in mice: A role for intraneuronal amyloid. Neurosci Biobehav Rev 31, 125-147.

[3] Blázquez G, Cañete T, Tobeña A, Giménez-Llort L, Fernández-Teruel A (2014) Cognitive and emotional profiles of aged Alzheimer's disease (3xTg-AD) mice: Effects of environmental enrichment and sexual dimorphism. Behav Brain Res 268, 185-201.

[4] Giménez-Llort L, Arranz L, Maté I, De la Fuente M (2008) Gender-specific neuroimmunoendocrine aging in a tripletransgenic 3xTgAD mouse model for Alzheimer's disease and its relation with longevity. Neuroimmunomodulation $\mathbf{1 5}$, 331-343.

[5] Hirata-Fukae C, Li HF, Hoe HS, Gray AJ, Minami SS, Hamada K, Niikura T, Hua F, Tsukagoshi-Nagai H, Horikoshi-Sakuraba Y, Mughal M, Rebeck GW, LaFerla FM, Mattson MP, Iwata N, Saido TC, Klein WL, Duff KE, Aisen PS, Matsuoka Y (2008) Females exhibit more extensive amyloid, but not tau, pathology in an Alzheimer transgenic model. Brain Res 1216, 92-103.

[6] García-Mesa Y, Colie S, Corpas R, Cristòfol R, Comellas F, Nebreda AR, Giménez-Llort L, Sanfeliu C (2016) Oxidative stress is a central target for physical exercise neuroprotection against pathological brain aging. J Gerontol A Biol Sci Med Sci 71, 40-49.

[7] Rae EA, Brown RE (2015) The problem of genotype and sex differences in life expectancy in transgenic AD mice. Neurosci Biobehav Rev 57, 238-251.

[8] Arranz L, De Castro NM, Baeza I, Giménez-Llort L, De la Fuente M (2011) Effect of environmental enrichment on the immunoendocrine aging of male and female triple-transgenic 3xTg-AD mice for Alzheimer's disease. J Alzheimers Dis 25, 727-737.

[9] Brown MA, Sampson EL, Jones L, Barron AM (2013) Prognostic indicators of 6-month mortality in elderly people with advanced dementia: A systematic review. Palliat Med 27, 389-400. 
[10] Komarova NL, Thalhauser CJ (2011) High degree of heterogeneity in Alzheimer's disease progression patterns. PLoS Comput Biol 7, e1002251.

[11] Mitchell SL, Miller SC, Teno JM, Kiely DK, Davis RB, Shaffer ML (2010) Prediction of 6-month survival of nursing home residents with advanced dementia using ADEPT vs hospice eligibility guidelines. JAMA 304, 1929-1935.

[12] Levy C, Kheirbek R, Alemi F, Wojtusiak J, Sutton B, Williams AR, Williams AJ (2015) Predictors of six-month mortality among nursing home residents: Diagnoses may be more predictive than functional disability. Palliat Med 18, 100-106.

[13] Zeng A, Song X, Dong J, Mitnitski A, Liu J, Guo Z, Rockwood K (2015) Mortality in relation to frailty in patients admitted to a specialized geriatric intensive care unit. J Gerontol A Biol Sci Med Sci 70, 1586-1594.

[14] van Dijk PT, Dippel DW, Habbema JD (1991) Survival of patients with dementia. J Am Geriatr Soc 39, 603-610.

[15] Reisberg B, Ferris SH, Franssen EH, Shulman E, Monteiro I, Sclan SG, Steinberg G, Kluger A, Torossian C, de Leon MJ, Laska E (1996) Mortality and temporal course of probable Alzheimer's disease: A 5-year prospective study. Int Psychogeriatry 8, 291-311.

[16] Bowen JD, Malter AD, Sheppard L, Kukull WA, McCormick WC, Teri L, Larson EB (1996) Predictors of mortality in patients diagnosed with probable Alzheimer's disease. Neurology 47, 433-439.

[17] Williams MM, Xiong C, Morris JC, Galvin JE (2006) Survival and mortality differences between dementia with Lewy bodies vs Alzheimer disease. Neurology 67, 1935-1941.

[18] Mölsä PK, Marttila RJ, Rinne UK (1995) Long-term survival and predictors of mortality in Alzheimer's disease and multi-infarct dementia. Acta Neurol Scand 91, 159-164.

[19] Agüero-Torres H, Fratiglioni L, Guo Z, Viitanen M, Winblad B (1998) Prognostic factors in very old demented adults: A seven-year follow-up from a population-based survey in Stockholm. J Am Ger Soc 46, 444-452.

[20] Larson EB, Shadlen MF, Wang L, McCormick WC, Bowen JD, Teri L, Kukull WA (2004) Survival after initial diagnosis of Alzheimer disease. Ann Int Med 140, 501-509.

[21] You EC, Dunt DR, White V, Vander Hoorn S, Doyle C (2014) Risk of death or hospital admission among community-dwelling older adults living with dementia in Australia. BMC Geriatry 14, 71.
[22] López-Pousa S, Olmo JG, Franch JV, Estrada AT, Cors OS, Nierga IP, Gelada-Batlle E (2006) Comparative analysis of mortality in patients with Alzheimer's disease treated with donepezil or galantamine. Age Ageing 35, 365-371.

[23] Shepperd S, Doll H, Angus RM, Clarke MJ, Iliffe S, Kalra L, Ricauda NA, Wilson AD (2008) Admission avoidance hospital at home. Cochrane Database Syst Rev 8, CD007491.

[24] Kilkenny C, Browne WJ, Cuthill IC, Emerson M, Altman DG (2010) Improving bioscience research reporting: The ARRIVE guidelines for reporting animal research. PLoS Biol 8, e1000412.

[25] Fahlström A, Yu Q, Ulfhake B (2011) Behavioral changes in aging female C57BL/6 mice. Neurobiol Aging 32, 18681880.

[26] Fahlström A, Zeberg H, Ulfhake B (2012) Changes in behaviors of male C57BL/6J mice across adult life span and effects of dietary restriction. Age (Dordr) 34, 1435-1452.

[27] Vida C, De la Fuente M (2013) Stress-related behavioural responses, immunity and ageing in animal models. In Immunosenescence: Psychosocial and behavioral determinants, Bosch JA, Phillips AD, Lord JM, eds. Springer, Science + Business Media NY, pp. 125-144.

[28] Baeta-Corral R, Giménez-Llort L (2014) Persitent hyperactivity and distinctive strategy features in the Morris water maze in 3xTg-AD mice at advanced stages of disease. Behav Neurosci 129, 129-137.

[29] Johnson IP (2015) Age-related neurodegenerative disease research needs aging models. Front Aging Neurosci 7, 168.

[30] Wallace LM, Howlett SE (2016) Commentary: Age-related neurodegenerative disease research needs aging models. Front Aging Neurosci 8, 9.

[31] Arranz L, Giménez-Llort L, De Castro NM, Baeza I, De la Fuente M (2009) Social isolation during old age worsens cognitive, behavioral and immune impairment. Rev Esp Geriatr Gerontol 44, 137-142.

[32] Giménez-Llort L, Torres-Lista V, De la Fuente M (2014) Crosstalk between behavior and immune system during the prodromal stages of Alzheimer's disease. Curr Pharm Des 20, 4723-4732.

[33] Giménez-Llort L, Mate I, Manassra RM, Vida C, De la Fuente M (2012) Peripheral immune system and neuroimmune communication impairment in a mouse model of Alzheimer's disease. Ann N Y Acad Sci 1262, 74-84. 